\title{
PROKLA-Redaktion
}

\section{Editorial: Soziale Kämpfe in Afrika}

Seit zwei Jahren erfahren wir über soziale Kämpfe auf dem afrikanischen Kontinent so viel wie seit den Unabhängigkeitsbewegungen nicht mehr: Mit dem „Arabischen Frühling“ sind politische Massenproteste im Maghreb und Maschrek in Europa zum prominenten Thema in Politik, Medien und Sozialwissenschaft avanciert. Zugleich fanden auch südlich der Sahara weitreichende Proteste statt, die von internationalen Debatten jedoch meist unbeachtet blieben. Streiks, Aufstände und andere Aktionen gab es in Benin, Botswana, Burkina Faso, der Côte d'Ivoire, Gabun, Kamerun, Kenia, Madagaskar, Malawi, Mauretanien, Mosambik, Namibia, Nigeria, Senegal, Simbabwe, Südafrika, Swaziland, Uganda und West-Sahara. Als das „Afrikanische Erwachen“ bezeichnet Firoze Manji, Herausgeber eines jüngst erschienenen gleichnamigen Sammelbands und des Nachrichtenportals Pambazuka News, die Proteste, die sich über den gesamten afrikanischen Kontinent erstrecken. ${ }^{1}$ Einige stellen wir in diesem Heft exemplarisch vor.

Die gegenwärtigen sozialen Kämpfe in Afrika sind vielfältig und beziehen sich auf zahlreiche Themenfelder: Wahlen und demokratische Rechte; Arbeitskämpfe und Ressourcenverteilung;

1 Firoze Manji (Hrsg.): African awakening: the emerging revolutions, Cape Town: Pambazuka, 2012.
"Strukturanpassung“, die Kommodifizierung öffentlicher Güter und sozialer Grundversorgung; Stadtentwicklung, Zugang zu Land und vieles mehr. Typische Bedingungen, welche die aktuellen gesellschaftlichen Auseinandersetzungen in vielen afrikanischen Staaten prägen, sind dabei die Spuren kolonialer Herrschaft, die in Politik, Wirtschaft und Verwaltung vielfach fortwirken, sowie der Einfluss von Internationalen (Finanz-)Institutionen. Gegenwärtig sind Sozialproteste vor allem in den Städten zu beobachten, was deutlich macht, dass auch der vergleichsweise geringe Urbanisierungsgrad in vielen afrikanischen Staaten bei gleichzeitig starkem Anwachsen einiger Städte sowie die ausgeprägte Armut und Ungleichheit innerhalb der städtischen Bevölkerungen eine nicht zu vernachlässigende Kontextbedingung darstellt. In diesem Zusammenhang fällt auf, dass bei einem relativ geringen Anteil industrieller Produktion an vielen afrikanischen Volkswirtschaften in den meisten Staaten Gewerkschaften dennoch einflussreiche Akteure sind - wobei die stärksten Gewerkschaften, wenig überraschend, oft aus dem öffentlichen Sektor (insbesondere den Bereichen Bildung und Gesundheit) kommen. Gleichzeitig ist ein großer Teil vor allem der armen städtischen Bevölkerung im informellen Sektor tätig und damit teils gewerkschaftlich (wenngleich nicht im Sinne von Groß-bzw. Industriegewerkschaften), teils 
in anderen Zusammenschlüssen (etwa Jugendverbände, Kooperativen, Frauen- oder Stadtteilgruppen) organisiert.

Die Proteste beispielsweise in Dakar (Senegal), wo ein breites Bündnis aus unterschiedlichen Gruppen gegen die Kandidatur von Abdoulaye Wade für eine dritte Amtszeit als Staatspräsident protestierte, wurden vor allem von der Jugendbewegung getragen (siehe dazu den Beitragvon Louisa Prause). Wade ließ sich zwar an der Kandidatur nicht hindern, verlor die Wahl aber schließlich gegen seinen Herausforderer Macky Sall. Gewerkschaften spielen in den meisten Auseinandersetzungen um die enorm gestiegenen Lebenshaltungskosten eine führende Rolle, die im Zuge der jüngsten Nahrungsmittelpreiskrise in zahlreichen afrikanischen Städten stattfinden, was Bettina Engels in ihrem Artikel untersucht. Auch in dem achttägigen Generalstreik gegen die Erhöhung des Benzinpreises in Nigeria Anfang 2012 war die Gewerkschaftsbewegung eine treibende Kraft (vgl. den Beitrag von Baba Aye). Dabei bewegen sich die meisten afrikanischen Gewerkschaften in einem Spannungsfeld zwischen der Vertretung von Mitgliederinteressen und übergreifendem gesellschaftspolitischen Anspruch. Mit dem Verhältnis von Gewerkschaften und sozialen Bewegungen befasst sich Carmen Ludwig am Beispiel Kapstadts. Dort bemüht sich die South African Municipal Workers' Union (SAMWU) um eine Zusammenarbeit mit lokalen Basisinitiativen im Bereich der Stadtentwicklungspolitik und der öffentlichen Versorgung. Ein nicht minder spannungsreiches Verhältnis steht im Mittelpunkt des Artikels von Nigel Gibson, der sich ebenfalls mit Südafrika in der Post-Apartheid-Ära befasst: die Bewegung der Shackbewohner_innen Abahlali baseMjondolo und ihr Verhältnis zum ANC.
Viele der Beiträge richten den Blick auf die Akteure aktueller gesellschaftlich-politischer Auseinandersetzungen in Afrika. Trotzdem geht es uns um soziale Kämpfe insgesamt und nicht (nur) um soziale Bewegungen. Für unser Verständnis sozialer Kämpfe (in Afrika wie anderswo) ist die Rückkopplung konkreter Auseinandersetzungen an politisch-ökonomische Strukturen auf unterschiedlichen Ebenen (lokal, national, global) von besonderer Relevanz.

Vier sich überlagernde historische Phasen sozialer Kämpfe in Afrika südlich der Sahara lassen sich seit Mitte des 20. Jahrhunderts identifizieren. ${ }^{2}$ Der Dekolonialisierung ab den 1950er Jahren mit den antikolonialen Befreiungsbewegungen als historisch bedeutende soziale Bewegungen, folgte die Phase der „Befreiungsbewegungen an der Macht“. In den frühen 1990er Jahren fand in vielen Staaten eine Transformation der politischen Systeme (insbesondere hin zu Mehrparteiensystemen) statt und große Hoffnungen wurden in das demokratische Potenzial der Zivilgesellschaften gesetzt. Dem schloss sich die Phase der Kooptierung eben jener Zivilgesellschaften durch internationale Akteure und Geber wie die Weltbank an. In dieser Phase gerieten viele afrikanische Staaten als Folge der Schuldenkrise unter den Druck der internationalen Finanzinstitutionen. Allerdings reagierten diese auch auf die Kritik sozialer Bewegungen der 1980er und 1990er Jahre und legten sogenannten Armutsreduzierungsstrategien vor, die dazu führten, dass sich eine beträchtliche Zahl zivilgesellschaftlicher Akteure in professionelle Dienstleister im Entwicklungsbereich

2 Brandes, Nikolai; Engels, Bettina (2011): Social Movements in Africa, in: Stichproben - Wiener Zeitschrift für kritische Afrikastudien, 11. Jg., Nr. 20, 1-15. 
verwandelten. Im Mittelpunkt dieses Heftes stehen demgegenüber soziale Kämpfe und Bewegungen, die auf eine grundlegende Veränderung bestehender Macht- und Herrschaftsverhältnisse abzielen.

Zweifelsohne kennzeichnen vielfältige historische, ökonomische, soziale und politische Strukturen und Prozesse die gegenwärtigen Gesellschaften in Afrika südlich der Sahara (ebenso wie jene in anderen Weltregionen auch), und eine pauschale Gegenüberstellung der Weltregionen mag analytisch mehr verschleiern als aufdecken. Die skizzierten historischen Makrotrends erweisen sich jedoch für die meisten afrikanischen Kontexte als einflussreich - und prägen damit die spezifischen Bedingungen, die soziale Kämpfe in Afrika von denen in anderen Weltregionen zugleich unterscheiden und mit ihnen verbinden. Denn die Geschichte Europas ist mit jener Afrikas und anderer ehemals kolonialisierten Weltregionen untrennbar verwoben. Dass dies für soziale Kämpfe ebenso gibt, zeigt sich unter den Bedingungen der gegenwärtigen globalen Krisen einmal mehr. Vor diesem Hintergrund untersucht ClausDieter König soziale Bewegungen in Westafrika mithilfe der Regulationstheorie. Er argumentiert, dass „das zwischenzeitlich gut funktionierende Zusammenwirken extravertierter kapitalistischer Akkumulation mit einer Regulation, die geprägt ist von der afrikanischen Spielweise des neoliberalen Staates und der Ausbreitung von Nichtregierungsorganisationen als Träger der Entwicklungszusammenarbeit, zunehmend in die Krise" gerät. Weniger optimistisch fällt hingegen das Fazit von Michael Watts' Analyse der Aktivitäten internationaler Öl-Unternehmen und des nigerianischen Staats aus, welche die sozial-ökologischen und politisch-ökonomischen Räume in der Nigerdelta-Region seit Jahrzehnten maßgeblich prägen. Watts stellt die damit verbundenen Kämpfe verschiedener Gruppen und Bewegungen um politische Autonomie, territoriale und Ressourcenkontrolle dar. Er kommt zu dem Schluss, dass ,sich die gewalttätige ethnisierte Akkumulation innerhalb des Öl-Komplexes in einer Gewaltökonomie selbst reproduzieren"kann.

Die enge Verzahnung von geostrategischen und sicherheitspolitischen Interessen mit Fragen der Rohstoffausbeutung tritt auch in der jüngsten Krise im westafrikanischen Mali deutlich zutage. Thomas Greven erläutert in seinem Beitrag die gesellschaftlichen und politischen Hintergründe der dortigen Rebellion und zeigt dabei, dass diese nur durch die Kombination innergesellschaftlicher und innerstaatlicher mit internationalen Einflüssen zu verstehen ist.

Außerhalb des Schwerpunkts gibt Dieter Boris einen Überblick über zentrale Veränderungen in der Sozialstruktur Lateinamerikas und Thomas Matys untersucht die Konsequenzen der zunehmenden (und erfolgreichen Versuche von Konzernen, Rechte zu beanspruchen, die bislang nur Personen hatten.

Dem Verein AfricAvenir danken wir, dass er uns die Übersetzung des Textes von Nigel Gibson zur Verfügung gestellt hat. Unser ganz besonderer Dank gilt unserer Gastredakteurin Bettina Engels. Sie hat nicht nur einen wichtigen Artikel beigesteuert, sie hat auch den Schwerpunkt betreut und maßgeblich gestaltet. Ohne ihr großes Engagement wäre dieses Heft nicht zustande gekommen.

Schließlich ist eine traurige Nachricht zu vermelden. Am 26. September 2012 verstarb Susanne Piening. Sie war Mitgründerin der PROKLA und unterstützte die Zeitschrift bis zu ihrem Tod als Mitglied im herausgebenden Verein, der „Vereinigung zur Kritik der politischen Ökonomie“. Wir werden sie vermissen. 